\title{
Clinical presentation and precipitating factors of diabetic ketoacidosis among patients admitted to intensive care unit at a tertiary hospital in Mwanza, Tanzania
}

\author{
SHABANI IDDI ${ }^{1 *}$, FRANCIS A. BRAYSON², HYASINTA JAKA ${ }^{2}$, MARIAM M. MIRAMBO 3 and MARTHA F. MUSHI ${ }^{3}$ \\ ${ }^{1}$ Department of Physiology, Weill Bugando School of Medicine, Catholic University of Health and Allied Sciences, \\ P.O. Box 1464, Mwanza, Tanzania \\ ${ }^{2}$ Department of Internal Medicine, Weill Bugando School of Medicine, Catholic University of Health and Allied \\ Sciences, Mwanza, Tanzania \\ 3Department of Microbiology/Immunology, Weill Bugando School of Medicine, Catholic University of Health and \\ Allied Sciences, Mwanza, Tanzania
}

\begin{abstract}
Background: Diabetic ketoacidosis (DKA), one of the common emergencies in patient with diabetes mellitus is associated with considerable morbidity and mortality. This study aimed to determine clinical presentation and precipitating factors of DKA among patients admitted at Bugando Medical Centre (BMC) in north-western Tanzania.

Methods: This study involved a retrospective review of hospital records of DKA patients admitted to intensive care unit at BMC during 2012. Data on demographics, precipitating factors, clinical presentation, duration of hospital admission and mortality were extracted and analysed.

Results: Total records of 1,906 hospitalized patients in 2012 were reviewed. Of this, 29 (1.5\%) had DKA. Of the 29 DKA patients, $18(62.1 \%)$ and 11 (37.9\%) were males and females, respectively. Among them 21(72.4\%) were known diabetics and $8(27.6 \%)$ were newly diagnosed to be diabetics. Twelve patients (41.1\%) presented with polyuria, polydipsia and general body malaise. Eleven (37.9\%) patients presented with loss of consciousness while 6(20.7\%), 4(13.8\%), 3(10.3\%) and 1(3.4\%) presented with vomiting, abdominal pain, Kussmaul's breathing and coma, respectively. Nausea, weight loss and polyphagia each were presented by $2(6.9 \%)$ patients. The precipitating factors were infection $15(51.7 \%)$, first presentation of diabetes mellitus 6 (20.7\%), missed insulin injection 6 (20.7\%) and co-morbid conditions 6 (20.7\%). Four (13.8\%), 1 (3.45\%) and $1(3.45 \%)$ had stroke, chronic renal failure and hypertension, respectively. Among the DKA patients, 22 (75.9\%) improved and were discharged, and 7 (24.1\%) died.

Conclusion: DKA occurred in about $1.5 \%$ of the patients admitted to ICU and it was a major cause of morbidity and mortality. The main precipitating factor was infection. Since the precipitating factors are preventable, health care providers should put emphasis in educating diabetic patients at the diabetes clinic to reduce morbidity and mortality in these patients.
\end{abstract}

Keywords: diabetic ketoacidosis, clinical presentation, precipitating factors, Tanzania

\section{Introduction}

Diabetic ketoacidosis (DKA), one of the common emergencies in patient with diabetes mellitus is associated with considerable morbidity and mortality (Levetan et al., 1997; Umpierrez \& Kitabchi, 2003). DKA is a complication of both type 1 diabetes mellitus (T1DM) and type 2 diabetes mellitus (T2DM). However, it is more commonly observed in type 1 (Smith et al., 1998; Umpierrez \& Kitabchi, 2003). In all diabetic patients presenting with DKA about one quarter will have type 2 diabetes and in patient presenting with DKA as a first manifestation of diabetes, about $15 \%$ will be type 2 (Newton \& Raskin, 2004). An epidemiologic study of hyperglycaemic states has shown that occurrence of DKA varied among different ethnic groups in Texas, USA (Balasubramanyam et al., 1999). Eighty percent of whites admitted with DKA were classified as having T1DM while only 53\% of African Americans and 34\% of Hispanic patients who were admitted with DKA had T1DM. Contrary to that finding, in Africa, DKA is encountered in $34 \%$ of African patients with type 2

\footnotetext{
* Correspondence Email: shabsizya2007@yahoo.co.uk
} 
diabetes (Sidibe, 2000) as a form of ketosis-prone diabetes that affects individuals in all age groups (Mbanya et al., 2010).

The incidence of DKA is difficult to establish, however, population based-studies in the USA recorded it to be ranging from 4.6 to 8 episodes per 1,000 patients with diabetes(Johnson et al., 1980). The frequency of DKA relative to newly diagnosed diabetes globally varies regionally from $11 \%$ to $67 \%$ in Europe (Levy-Marchal et al., 2001) to $28.4 \%$ in the USA (Rosenbloom, 2010a,b). The true incidence of DKA in tropical Africa is unknown but has been estimated at $24 \%$ (IDF, 2011, suggesting that many cases are underreported or misdiagnosed. The severity of acute diabetic complication can be appreciated from high death-case ratio of 5-10\% (Faich et al., 1983). In Africa the mortality rate of DKA is unacceptably higher (26\%-29\%) as reported in studies from Kenya, Tanzania and Ghana (McLarty et al., 1996; Mbugua et al., 2005; Otieno et al., 2005; Majaliwa et al., 2007).

Most frequent presenting complaint include vomiting, abdominal pain, dehydration and respiratory distress (Balasubramanyam et al., 1999). On physical examination, may present with signs of dehydration, tachycardia and hypotension (Faich et al., 1983). Patients with DKA may present with varying degrees of levels of consciousness, majority being alert, and less than onefifth being comatose (Otieno et al., 2005). Patients with severe metabolic acidosis will usually present with a distinctive rapid and deep breathing (Kussmaul's breathing) (Faich et al., 1983). Various risk factors have been reported to precipitate DKA in diabetic patients. They include infections, non-compliance to treatment and co-morbid states like stroke, chronic renal failure, chronic liver disease and acute pancreatitis (Mallare et al., 2003; Lin et al., 2005).

Although DKA is potentially life threatening, it is entirely a preventable diabetic complication. There is paucity of data on diabetic ketoacidosis in Tanzania. It is important to document the clinical presentation and precipitating factors of DKA in order to provide measure to prevent DKA and its associated morbidity and mortality. The present study was carried out to determine the clinical presentation and precipitating factors of DKA among patients admitted to intensive care unit at Bugando Medical Centre in north-western Tanzania.

\section{Materials and Methods}

\section{Study area and data collection}

This descriptive retrospective hospital based cross sectional study was conducted at Bugando Medical Centre in Mwanza, Tanzania. Bugando Medical Centre is the consultant, referral and teaching hospital for the Lake Victoria and western zones of Tanzania, which include six regions of Mwanza, Mara, Kigoma, Shinyanga, Tabora, and Kagera.

All diabetic patients admitted to the intensive care unit (ICU) ward at Bugando Medical Centre (BMC) in 2012 were included in the study. All patients who met the inclusion criteria were recruited for the study by random sampling technique. A total of 1,906 files for the year 2012 were reviewed randomly and all patients with confirmed DKA were included in the study. A data collection sheet (checklist) was used to extract information or data from the patient's files and registers including data on demographics, precipitating factors, clinical presentation, duration of hospital admission and mortality.

\section{Data analysis}

Data were analysed using Epidata 3.1 and Statistical Package for Social Science (SPSS) 17.0 computer programmes. Data were summarized into proportions and presented in tables.

\section{Ethical consideration.}

The study received ethical clearance from the Joint Catholic University of Health and Allied Science/Bugando Medical Centre Ethics Review Committee. Permission to collect data from the files and registers was granted by the Medical Record Department of Bugando Medical Centre. 


\section{Results}

Of the total 1,906 patients, 29 (1.5\%) diagnosed with DKA patients were involved in this study. Among 29 patients with DKA 19 (65.5\%) were above 30 years, 4 (13.8\%) were between 1 to 10 years, $3(10.3 \%)$ were $11-20$ and $21-30$ years. Among them 18 (62.1\%) were males and 11 (37.9\%) were females. Among 29 patients who presented with DKA, 21 (72.4\%) were known diabetic patients and $8(27.6 \%)$ were newly diagnosed diabetes mellitus patients. Of the 29 DKA patients, the majority presented with polyuria, polydipsia and general body malaise. Other clinical presentations were loss of consciousness, vomiting, abdominal pain, Kussmaul's breathing, nausea, weight loss, polyphagia and coma. (Table1).

Table 1: Clinical presentations among diabetic ketoacidosis patients $(n=29)$

\begin{tabular}{lcl}
\hline Clinical presentation & Number of patients & Percentage \\
\hline Nausea & 2 & 6.9 \\
Vomiting & 6 & 20.7 \\
Polyuria & 12 & 41.4 \\
Polydipsia & 12 & 41.4 \\
Abdominal pain & 4 & 13.8 \\
Weight loss & 2 & 6.9 \\
Polyphagia & 2 & 6.9 \\
Loss of consciousness & 11 & 37.9 \\
General body malaise & 12 & 41.4 \\
Kussmaul's breathing & 3 & 10.3 \\
Confusion & 2 & 6.9 \\
Blurred vision & 0 & 0 \\
Coma & 1 & 3.4 \\
\hline
\end{tabular}

The main precipitating factor was infection. Other precipitating factors were first presentation of diabetes mellitus, missed insulin injection and co-morbid conditions (including stroke, chronic renal failure and hypertension) (Table 2). None of the patients presented with myocardial infarction, peripheral vascular disease, gangrene and chronic renal failure.

Table 2: Diabetic ketoacidosis precipitating factors $(\mathrm{n}=\mathbf{2 9})$

\begin{tabular}{lcl}
\hline Precipitating factor & Number & Percentage \\
\hline Infection & 15 & 51.7 \\
First presentation of diabetes mellitus & 6 & 20.7 \\
Missed insulin injection & 6 & 20.7 \\
Co-morbidity & 6 & 20.7 \\
Stroke & 4 & 13.8 \\
Chronic renal failure & 1 & 3.4 \\
Hypertension & 1 & 3.4 \\
\hline
\end{tabular}

Sixteen patients (55.2\%) spent 1 to 5 days in the ICU, while the rest spent more than six days. Majority of the patients, 22 (75.9\%) improved and were discharged, and 7 (24.1\%) died.

\section{Discussion}

In the present study most of the patients were above 30 years of age. This finding is consistent with the previous studies (Umpierrez et al. 1997, 1999). More than half of our study subjects were known diabetics while only about a quarter were newly diagnosed. This is different as compared to the finding of the previous studies in Nigeria and Kenya which showed more than half DKA patients to be newly diagnosed (Mbugua et al., 2005; Edo, 2012). Although DKA is potentially life threatening, it is entirely a preventable diabetic complication. However, finding of large number of 
known diabetes mellitus patients presented with signs and symptoms of DKA admitted to ICU in the current study may be due to inadequacy or lack of training of health professionals, lack of public awareness as well as lack of health education to individual patients/families among others (Majaliwa et al., 2010).

Among the precipitating factors, infection was the most presented precipitating factor followed by first presentation of diabetes mellitus. This is similar to the findings of the previous study in Nigeria (Edo, 2012) and contrary to the findings of a study in Kenya (Mbugua et al., 2005). The difference in the findings of the present study and that in Kenya (Mbugua et al. 2005) could be due to lack of adequate knowledge on diabetes and its precipitants, or simply the omission or failure to take the appropriate dosage especially during illnesses (Umpierrez et al., 1999). This explanation is supported by observation that more of our patients with DKA where known diabetes mellitus patients and therefore might have already been exposed to diabetes education.

Our finding on clinical presentation is contrary to the finding of the study in Kenya by Mbugua et al. (2005), which showed more than 90\% had altered level of consciousness, with almost a quarter in coma, one-third had systolic hypotension, and almost three-quarters had moderate to severe dehydration. Three-quarters of our patients improved and were discharged home. However the mortality rate among our patients was higher than that reported in a study in Nigeria (Edo, 2012).

In conclusion, although DKA accounted for less than two percent of the patients admitted to ICU at Bugando Medical Centre, it was the major cause of morbidity and mortality. The main precipitating factor was infection. Since the main precipitating factors are preventable, health care providers should put emphasis in educating diabetic patients at the diabetes clinic to reduce morbidity and mortality in these patients.

\section{Acknowledgements}

We would like to acknowledge the assistance and guidance provided by all staff members of the Department of Internal Medicine and medical records department, Bugando Medical Centre. This research was supported by Catholic University of Health and Allied Sciences, Bugando.

\section{Competing interests}

The authors declare that they have no competing interest.

\section{Author's contribution}

SI, BFA designed the study, collected and analysed data. HJ conceived the study and coordination data collection. SI, MFM, HJ, MMM drafted and critically revised the manuscript. All authors read and approved the final draft of the manuscript.

\section{References}

Balasubramanyam, A., Zern, J.W., Hyman, D.J. \& Pavlik, V. (1999) New profiles of diabetic ketoacidosis: type 1 vs type 2 diabetes and the effect of ethnicity. Archives of Internal Medicine 159: 2317-2322.

Edo, A. E. (2012) Clinical profile and outcomes of adult patients with hyperglycemic emergencies managed at a tertiary care hospital in Nigeria. Nigerian Medical Journal 53: 121.

Faich, G. A., Fishbein, H. A. \& Ellis, S. E. (1983). The epidemiology of diabetic acidosis: a populationbased study. American Journal of Epidemiology, 117: 551-558.

IDF (2011) Diabetes Atlas 5th ed. International Diabetes Federation. Brussels, Belgium. 
Johnson, D., Palumbo, P. \& Chu, C. (1980) Diabetic ketoacidosis in a community-based population. Mayo Clinic Proceedings 83-88pp.

Levetan, B., Levitt, N. \& Bonnici, F. (1997) Hyperglycaemic emergencies are a common problem. South African Medical Journal 87: 368-370.

Levy-Marchal, C., Patterson, C., Green, A. \& Group, E.A.S. (2001) Geographical variation of presentation at diagnosis of type I diabetes in children: the EURODIAB study. Diabetologia 44: B75-B80.

Lin, S.-F., Lin, J.-D. \& Huang, Y.-Y. (2005) Diabetic ketoacidosis: comparisons of patient characteristics, clinical presentations and outcomes today and 20 years ago. Chang Gung Medical Journal 28: 24-30.

Majaliwa, E., Mohn, A., Chiavaroli, V., Ramaiya, K., Swai, A. \& Chiarelli, F. (2010) Managment of diabetic ketoacidosis in children and adolescents in sub-Saharan Africa: a review. East African Medical Journal 87: 167-173.

Majaliwa, E.S., Munubhi, E., Ramaiya, K., Mpembeni, R., Sanyiwa, A., Mohn, A. \& Chiarelli, F. (2007) Survey on acute and chronic complications in children and adolescents with type 1 diabetes at Muhimbili National Hospital in Dar es Salaam, Tanzania. Diabetes Care 30: 2187-2192.

Mallare, J.T., Cordice, C.C., Ryan, B.A., Carey, D.E., Kreitzer, P.M. \& Frank, G.R. (2003) Identifying risk factors for the development of diabetic ketoacidosis in new onset type 1 diabetes mellitus. Clinical Pediatrics 42: 591-597.

Mbanya, J. C.N., Motala, A.A., Sobngwi, E., Assah, F.K. \& Enoru, S.T. (2010) Diabetes in sub-Saharan Africa. Lancet 375: 2254-2266.

Mbugua, P., Otieno, C., Kayima, J., Amayo, A. \& Mcligeyo, S. (2005) Diabetic ketoacidosis: clinical presentation and precipitating factors at Kenyatta National Hospital, Nairobi. East African Medical Journal 82: S191-196.

Mclarty, D., Unwin, N., Kitange, H. \& Alberti, K. (1996) Diabetes mellitus as a cause of death in SubSaharan Africa: results of a community-based study in Tanzania. Diabetic Medicine 13: 990995.

Newton, C.A. \& Raskin, P. (2004) Diabetic ketoacidosis in type 1 and type 2 diabetes mellitus: clinical and biochemical differences. Archives of Internal Medicine 164: 1925-1931.

Otieno, C., Kayima, J., Omonge, E. \& Oyoo, G. (2005). Diabetic ketoacidosis: risk factors, mechanisms and management strategies in sub-Saharan Africa: a review. East African Medical Journal 82: S197-203.

Rosenbloom, A.L. (2010a) Hyperglycemic hyperosmolar state: an emerging pediatric problem. The Journal of Pediatrics 156: 180-184.

Rosenbloom, A. L. (2010b) The management of diabetic ketoacidosis in children. Diabetes Therapy 1: $103-120$.

Sidibe, E. (2000) Main complications of diabetes mellitus in Africa. Annales de Medecine Interne 151: 624-628.

Smith, C., Firth, D., Bennett, S., Howard, C. \& Chisholm, P. (1998) Ketoacidosis occurring in newly diagnosed and established diabetic children. Acta Paediatrica 87: 537-541.

Umpierrez, G.E., Kelly, J.P., Navarrete, J.E., Casals, M.M. \& Kitabchi, A.E. (1997) Hyperglycemic crises in urban blacks. Archives of Internal Medicine 157: 669-675.

Umpierrez, G.E. \& Kitabchi, A. E. (2003) Diabetic ketoacidosis. Treatments in Endocrinology 2: 95108.

Umpierrez, G.E., Woo, W., Hagopian, W.A., Isaacs, S.D., Palmer, J.P., Gaur, L.K., Nepom, G.T., Clark, W.S., Mixon, P.S. \& Kitabchi, A.E. (1999) Immunogenetic analysis suggests different pathogenesis for obese and lean African-Americans with diabetic ketoacidosis. Diabetes Care 22: 1517-1523. 